\title{
Long-term tumor control following stereotactic radiosurgery for jugular paraganglioma using 3D volumetric segmentation
}

\author{
Neil S. Patel, MD, ${ }^{1}$ Matthew L. Carlson, MD, , ${ }^{1,2}$ Bruce E. Pollock, MD,, ${ }^{2,3}$ Colin L. W. Driscoll, MD,, \\ Brian A. Neff, MD, ${ }^{1}$ Robert L. Foote, MD, ${ }^{3}$ Christine M. Lohse, MS, ${ }^{4}$ and Michael J. Link, MD ${ }^{1,2}$ \\ Departments of ${ }^{1}$ Otorhinolaryngology, ${ }^{2}$ Neurologic Surgery, ${ }^{3}$ Radiation Oncology, and ${ }^{4}$ Health Sciences Research, Mayo Clinic, \\ Rochester, Minnesota
}

OBJECTIVE The morbidity of gross-total resection of jugular paraganglioma (JP) is often unacceptable due to the potential for irreversible lower cranial neuropathy. Stereotactic radiosurgery (SRS) has been used at the authors' institution since 1990 for the treatment of JP and other benign intracranial tumors. Conventional means of assessing tumor progression using linear measurements or elliptical approximations are imprecise due to the irregular shape and insinuating growth pattern of JP. The objective of this study was to assess long-term tumor control in these patients by using sliceby-slice 3D volumetric segmentation of serial MRI data.

METHODS Radiographic data and clinical records were reviewed retrospectively at a single, tertiary-care academic referral center for patients treated from 1990 to 2017. Volumetric analyses by integration of consecutive tumor crosssectional areas (tumor segmentation) of serial MRI data were performed. Tumor progression was defined as volumetric growth of $15 \%$ or greater over the imaging interval. Primary outcomes analyzed included survival free of radiographic and clinical progression. Secondary outcomes included new or worsened cranial neuropathy.

RESULTS A total of 85 patients were treated with Gamma Knife radiosurgery (GKRS) for JP at the authors' institution over the last 27 years. Sixty patients had pretreatment and serial posttreatment contrast-enhanced MRI follow-up suitable for volumetric analysis. A total of 214 MR images were analyzed to segment tumor images in a slice-by-slice fashion to calculate integral tumor volume. The median follow-up duration was 66 months (range 7-202 months). At 5 years the tumor progression-free survival rate was $98 \%$. Three tumors exhibited progression more than 10 years after GKRS. Estimated survival free of radiographic progression rates (95\% confidence interval [Cl]; $n=$ number still at risk) at 5, 10, and 15 years following radiosurgery were $98 \%$ (95\% Cl 94\%-100\%; $n=34), 94 \%(95 \% \mathrm{Cl} 85 \%-100 \% ; n=16)$, and $74 \%$ $(95 \% \mathrm{Cl} 56 \%-98 \% ; n=6)$, respectively. One patient with tumor progression required treatment intervention using external beam radiation therapy, constituting the only case of clinical progression. Two patients (3\%) without preexisting lower cranial nerve dysfunction developed new ipsilateral vocal fold paralysis following radiosurgery.

CONCLUSIONS SRS achieves excellent long-term tumor control for JP without a high risk for new or worsened cranial neuropathy when used in primary, combined modality, or recurrent settings. Long-term follow-up is critical due to the potential for late radiographic progression (i.e., more than 10 years after SRS). As none of the patients with late progression have required salvage therapy, the clinical implications of this degree of tumor growth have yet to be determined.

https://thejns.org/doi/abs/10.3171/2017.10.JNS17764

KEYWORDS stereotactic radiosurgery; glomus jugulare; paraganglioma; Gamma Knife radiosurgery; skull base

$\mathbf{J}$ UGULAR paragangliomas (JPs), also commonly referred to as glomus jugulare tumors, arise from paraganglia on the superior surface of the jugular bulb within the jugular foramen. These histologically benign tumors typically exhibit indolent growth within the temporal bone with potential to infiltrate the facial and/or lower cranial nerves (CNs), petrous carotid canal and/or artery, otic capsule, and posterior fossa. Early symptoms may be as subtle as pulsatile tinnitus or conductive hearing loss. With progressive tumor growth, dysphagia, dysphonia, and tongue

ABBREVIATIONS $\mathrm{Cl}=$ confidence interval; $\mathrm{CN}=$ cranial nerve; EBRT = external beam radiation therapy; $\mathrm{GKRS}=$ Gamma Knife radiosurgery; GTR = gross-total resection; ICA = internal carotid artery; IQR = interquartile range; JP = jugular paraganglioma; LINAC = linear accelerator; NTR = near-total resection; SRS = stereotactic radiosurgery; STR = subtotal resection.

SUBMITTED March 26, 2017. ACCEPTED October 2, 2017.

INCLUDE WHEN CITING Published online April 13, 2018; DOI: 10.3171/2017.10.JNS17764. 
weakness may develop as manifestations of lower $\mathrm{CN}$ involvement. Additionally, patients may develop headache, ataxia, or vomiting from elevated intracranial pressure from venous sinus thrombosis, or rarely, obstructive hydrocephalus. ${ }^{47}$ As many as $10 \%$ of JPs may be familial, inherited in an autosomal dominant pattern with paternal genomic imprinting. ${ }^{30,42}$ All JPs are highly vascular and develop within close proximity to the pars nervosa of the jugular foramen, rendering gross-total resection (GTR) challenging with a relatively high risk of lower $\mathrm{CN}$ injury. In light of this, less invasive treatment modalities have gained popularity to reduce morbidity.

External beam radiation therapy (EBRT) using orthovoltage, megavoltage, and cobalt-60 techniques increased in utilization from the 1950s through the 1980s. With surgery still considered the mainstay of JP therapy, EBRT was reserved for poor surgical candidates, elderly patients, or those with recurrent or enlarging residual tumors. Orthovoltage radiation (e.g., 200-500 kV x-ray sources) predictably resulted in a high rate of osteoradionecrosis of the temporal bone with unpredictable tumor control due to a higher absorption of the dose in bone and a lower dose delivered to the JP. Megavoltage radiotherapy using modern linear accelerators (LINACs) with higher energy and more deeply penetrating x-rays allowed the delivery of a higher dose to the JP and lower dose to bone, resulting in tumor control rates in the $61 \%-99 \%$ range. ${ }^{4-7,17,20,27}$ Based on these results, prior skepticism regarding the radiosensitivity of JP was quelled, leading to the eventual use of hypofractionated EBRT and stereotactic radiosurgery (SRS) for the primary treatment or treatment of recurrent JP.

The Gamma Knife (Elekta AB), CyberKnife (Accuray), and Novalis (BrainLAB) platforms have been used for the treatment of JP and other benign intracranial tumors since the 1960s. Several groups have reported series with excellent tumor control outcomes, for both primary $y^{3,8,12,15,16,22,32,33,38,39}$ and recurrent ${ }^{3,12,15,16,32,38,40} \mathrm{JP}$, with preservation of lower $\mathrm{CN}$ function. The purpose of this report is to describe a single-center experience treating JP with Gamma Knife radiosurgery (GKRS) since 1990. Unique to this series is serial measurement of tumor size using 3D tumor segmentation to provide an accurate assessment of tumor progression that avoids error introduced by volume approximation formulae or linear measurements. Three-dimensional volumetric segmentation is particularly advantageous for JP, where the amorphous and infiltrative growth pattern renders other methods imprecise.

\section{Methods \\ Data Collection}

Following Mayo Clinic IRB approval, a retrospective review of paper and electronic medical records for all patients with a diagnosis of JP treated with GKRS was performed. Diagnosis was established based on patient history, physical examination, imaging findings, and histopathology results (where available).

Clinical and operative notes as well as pathology and imaging reports were reviewed to obtain the follow- ing demographic data: age at radiosurgery, sex, laterality, prior treatment, diagnosis of familial paraganglioma, and $\mathrm{CN}$ function. Prior surgery included biopsy, subtotal resection (STR), near-total resection (NTR), and GTR. Radiation delivery parameters included marginal dose, maximum dose, volume treated, and number of isocenters. Maximum radiosurgical dose to the internal carotid artery (ICA), maximum cochlear dose, and mean cochlear dose were measured using the dose measurement tool in the GammaPlan software (Elekta $\mathrm{AB}$ ). Lower $\mathrm{CN}$ function was examined with flexible fiberoptic or mirror laryngoscopy. Facial nerve function was graded using the House-Brackmann grading system. ${ }^{21}$

\section{GKRS Treatment Parameters}

All patients were treated using the Leksell Gamma Knife (models U, B, G, or Perfexion, depending on year of treatment) at our institution. Treatment parameters are reported in Table 1. Median marginal and maximum doses were 16 and $32 \mathrm{~Gy}$, respectively. The median treated tumor volume was $11.6 \mathrm{~cm}^{3}$ (range $2.0-34.2 \mathrm{~cm}^{3}$ ) using a median of 10 isocenters (range 1-21 isocenters). As there were no large published series of JPs treated with single-fraction GKRS when we began treating these tumors, we used doses similar to how meningiomas of comparable volume were managed. As we gained experience and realized good tumor control with minimal morbidity, we continued using marginal doses of 15-16 Gy. Additionally, despite the relatively large volumes treated, because most of the radiation fall-off occurs in the skull base and subcranial soft tissues, we felt comfortable with these dose prescriptions limiting collateral injury to critical structures, such as the brainstem.

\section{Imaging Measurements}

Tumor volume measurements were performed using Aquarius iNtuition Edition (version 4.4.11, TeraRecon Inc.). Postgadolinium T1-weighted MRI sequences were loaded into the TeraRecon server from the institutional medical imaging database for analysis. Slice thickness varied from 1 to $5 \mathrm{~mm}$ based on radiological technique but was kept consistent within the same patient. Regions of interest (i.e., tumor cross-sectional area) were outlined in two dimensions on each individual axial slice. A volume of interest was then automatically computed by integrating each axial area across the craniocaudal dimension (Fig. 1). The MRI obtained immediately prior to GKRS treatment was used to calculate the baseline initial tumor volume. Serial posttreatment scans were ana-

TABLE 1. Summary of SRS treatment parameters in 60 patients with JP and appropriate imaging

\begin{tabular}{lc}
\hline \multicolumn{1}{c}{ Feature } & Median (IQR; range) \\
\hline Maximal dose in Gy & $32(31-33.8 ; 30-40)$ \\
\hline Marginal dose in Gy & $15.75(15-16 ; 12-18)$ \\
\hline Isodose line in \% & $50(0.45-0.5 ; 0.4-0.5)$ \\
\hline Initial vol in $\mathrm{mm}^{3}$ & $11,550(8875-16,398 ; 2000-34,200)$ \\
\hline No. of isocenters & $10(7-13 ; 1-21)$ \\
\hline
\end{tabular}



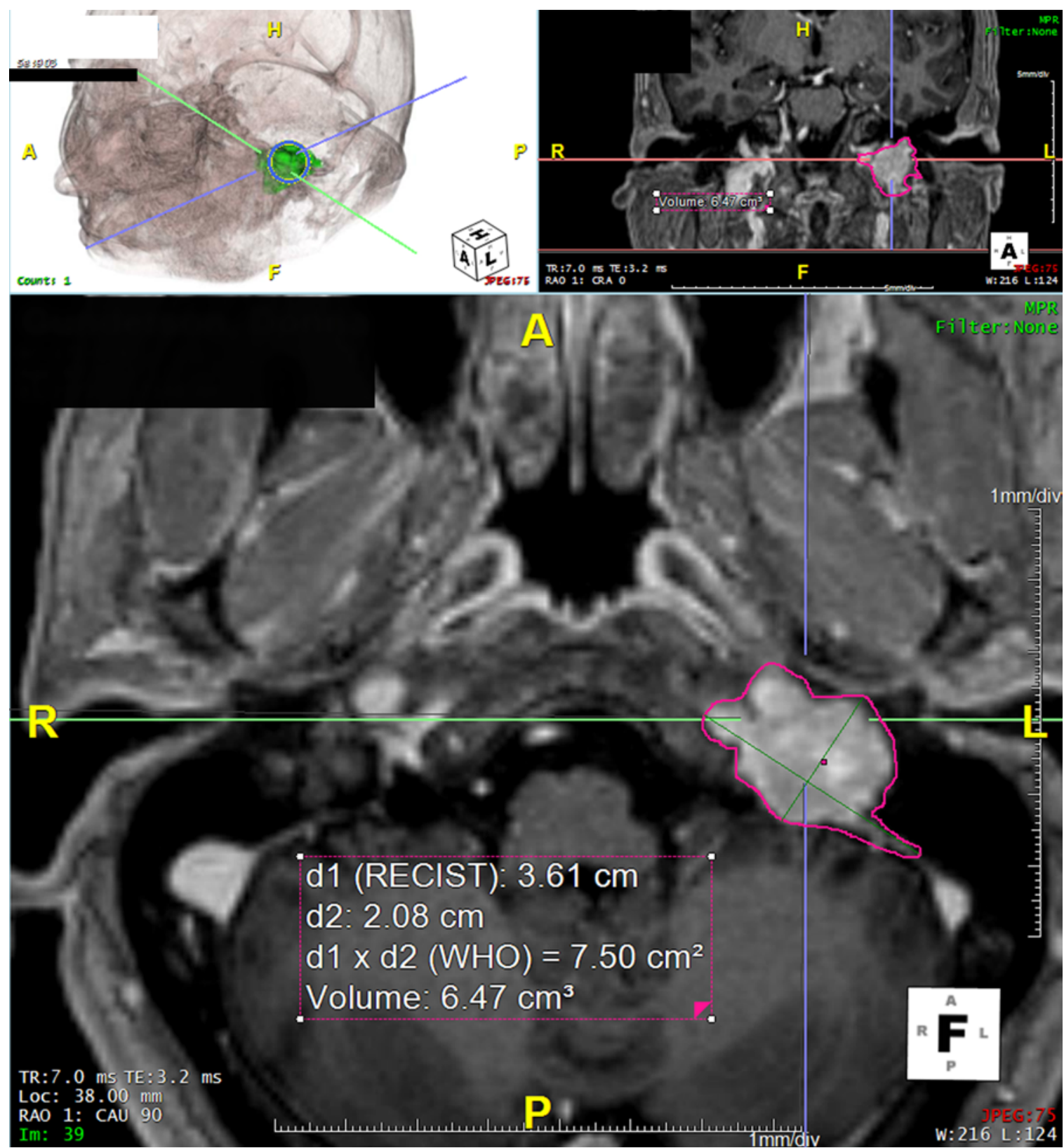

FIG. 1. Example of volumetric tumor analysis. While visualizing multiplanar MRI reconstructions, the tumor is outlined on each slice, a volume of interest is generated (upper left), and an integral volume is subsequently calculated. Figure is available in color online only.

lyzed using the same technique. The most recent study was always included for analysis to mark the endpoint of radiographic follow-up. Radiographic progression was considered present if volumetric growth of $15 \%$ or greater over the imaging interval was observed based on literature summarized in the discussion section. A random sample of 10 MR images was used to test consistency in the measurement method and verify that changes in volume were attributable to actual disease progression and not error induced by the rater. Each study was examined twice in random order. Pearson intrarater correlation coefficient was 0.994 ( $\mathrm{p}<0.01)$, demonstrating excellent agreement. A case in which tumor progression neces- sitated treatment intervention was considered a clinical progression.

\section{Statistical Analysis}

Continuous features were summarized with medians, interquartile ranges (IQRs), and ranges; categorical features were summarized with frequency counts and percentages. Survival free of radiographic progression was estimated using the Kaplan-Meier method, with the duration of followup calculated from the date of GKRS to the date of last radiographic follow-up. Statistical analyses were performed using the SAS software package (version 9.4, SAS Institute). 
TABLE 2. Summary of baseline features according to tumor growth

\begin{tabular}{|c|c|c|c|}
\hline \multirow[b]{2}{*}{ Feature } & \multicolumn{3}{|c|}{ Tumor Growth } \\
\hline & $15 \%$ or More $(n=5)$ & $2 \%-13 \%(n=14)$ & None $(n=41)$ \\
\hline Age at radiosurgery in $\mathrm{yrs}^{*}$ & $65(34-72 ; 32-75)$ & $56.5(40-68 ; 33-82)$ & $54(46-62 ; 18-85)$ \\
\hline Initial vol in $\mathrm{mm}^{3 *}$ & $5670(4148-5770 ; 2990-15,200)$ & 6965 (5777-8640; 3890-14,300) & $7180(5670-9710 ; 1370-20,700)$ \\
\hline Maximal dose in Gy* & $33(32-34 ; 32-34)$ & $32(32-33 ; 30-38)$ & $32(31-33 ; 30-40)$ \\
\hline Marginal dose in $\mathrm{Gy}^{*}$ & $16(16-17 ; 15-17)$ & $15(15-16 ; 13-17)$ & $16(15-16 ; 12-18)$ \\
\hline \multicolumn{4}{|l|}{ Sex, no. $(\%)$} \\
\hline Female & $3(60)$ & $10(71)$ & $27(66)$ \\
\hline Male & $2(40)$ & $4(29)$ & $14(34)$ \\
\hline \multicolumn{4}{|l|}{ Side, no. (\%) } \\
\hline Lt & $3(60)$ & $9(64)$ & $24(59)$ \\
\hline $\mathrm{Rt}$ & $2(40)$ & $5(36)$ & $17(41)$ \\
\hline Familial, no. (\%) & 0 & $2(14)$ & $5(12)$ \\
\hline Prior surgery, no. (\%) & $1(20)$ & $6(43)$ & $18(44)$ \\
\hline \multicolumn{4}{|l|}{ Type of prior surgery, no. (\%)† } \\
\hline STR & $1(100)$ & $2(33)$ & $9(56)$ \\
\hline GTR & 0 & $3(50)$ & $5(31)$ \\
\hline NTR & 0 & 0 & $1(6)$ \\
\hline Biopsy & 0 & $1(17)$ & $1(6)$ \\
\hline
\end{tabular}

* Median (IQR; range).

$\dagger$ In 23 patients total.

\section{Results}

\section{Tumor Control}

A total of 85 patients with JP have been treated with GKRS at the authors' institution since 1990. Sixty patients (70.6\%) had serial MRI with appropriate pre- and posttreatment gadolinium-enhanced T1-weighted sequences available for analysis. While the remainder of the patients ( $n=25,29 \%$ ) were followed radiographically, imaging studies were not available in the electronic record. The decision to intervene in patients treated with primary GKRS was primarily driven by symptoms, patient preference, and the goal of preventing eventual neurological complications. As most tumors were moderately large at the time of diagnosis (median tumor volume $=11.6 \mathrm{~cm}^{3}$ ) we believed allowing even minor additional growth could precipitate disabling symptoms that would be hard to reverse with either surgery or GKRS, and/or only increase the eventual risk of GKRS if the tumors were allowed to get bigger prior to treatment. In patients treated with secondary GKRS following prior resection $(n=25,42 \%)$ or observation $(\mathrm{n}=3,5 \%)$, the indication for treatment was tumor growth. The cumulative and median follow-up duration was 400 patient-years and 66 months (range 7-202 months), respectively. A total of 214 MRI studies, with a median of 4 studies per patient, were analyzed. Baseline features collected for the 60 patients with JP treated with GKRS between May 1991 and November 2015 are summarized in Table 2. Five patients experienced radiographic progression at $1.5,7.7,11.1,11.5$, and 11.9 years following radiosurgery. The median duration of follow-up for the 55 patients who did not experience progression was 5.3 years (IQR 1.9-9.5 years, range 0.6-22.3 years). Esti- mated rates of survival free of radiographic progression ( $95 \%$ confidence interval $[\mathrm{CI}] ; \mathrm{n}=$ number still at risk) at 5,10 , and 15 years following radiosurgery were $98 \%(95 \%$ CI $94 \%-100 \% ; \mathrm{n}=34), 94 \%(95 \%$ CI $85 \%-100 \% ; \mathrm{n}=16)$, and $74 \%(95 \%$ CI 56\%-98\%; n=6), respectively (Fig. 2). Associations with time to radiographic progression could not be evaluated because only 5 patients experienced the outcome of interest, and at least 10 are needed to support a statistical assessment. Instead, baseline features were summarized for the 5 patients who experienced progression (defined as $15 \%$ or more growth), the 14 who experienced a lesser degree of growth (2 to 13\%), and the 41 who did not demonstrate growth (Table 3). Individual tumor volumes over time are depicted graphically in Fig. 3.

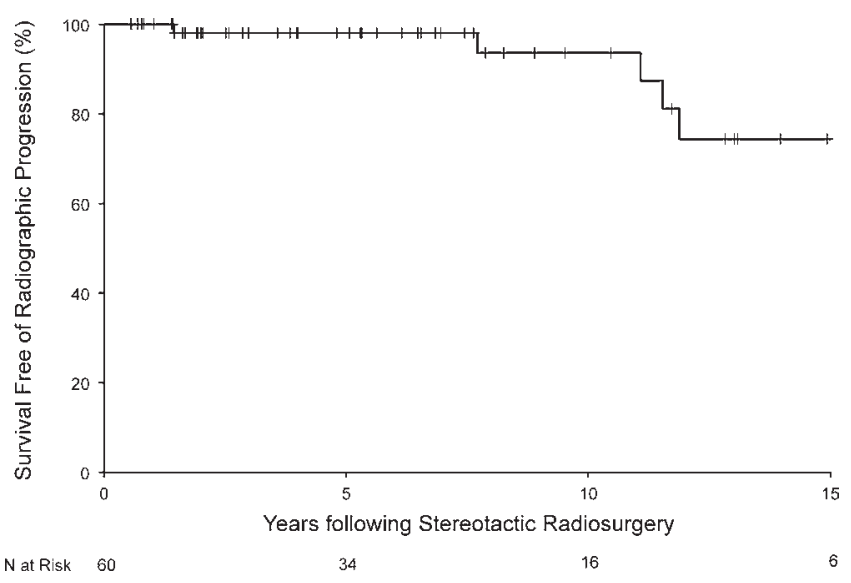

FIG. 2. Survival free of radiographic progression using the Kaplan-Meier method. 
TABLE 3. Summary of baseline features

\begin{tabular}{lc}
\hline \multicolumn{1}{c}{ Feature } & Value \\
\hline Age at radiosurgery in yrs* & $54.5(40.5-64.5 ; 18-85)$ \\
\hline Initial vol in mm $^{3 *}$ & $6855(5290-9270 ; 1370-20,700)$ \\
\hline Maximal dose in Gy* & $32(31-34 ; 30-40)$ \\
\hline Marginal dose in Gy* & $16(15-16 ; 12-18)$ \\
\hline Sex, no. (\%) & $40(67)$ \\
\hline Female & $20(33)$ \\
\hline Male & $36(60)$ \\
\hline Side, no. (\%) & $24(40)$ \\
\hline Lt & $7(12)$ \\
\hline Rt & $25(42)$ \\
\hline Familial, no. (\%) & \\
\hline Prior surgery, no. (\%) & $12(52)$ \\
\hline Type of prior surgery, no. (\%)† & $8(35)$ \\
\hline STR & $2(9)$ \\
\hline GTR & $1(4)$ \\
\hline Biopsy & \\
\hline NTR & \\
\hline
\end{tabular}

${ }^{*}$ Median (IQR; range).

$\dagger$ In 23 patients total.

\section{Decision to Intervene}

There were 35 patients treated with primary GKRS. Of these 35 patients, 25 (71\%) elected to undergo treatment with the intent to protect existing normal $\mathrm{CN}$ function and prevent tumor growth. Four patients (11\%) had a contralateral carotid body tumor or glomus vagale that threatened CN X function, and elected to undergo GKRS to protect ipsilateral CN X function. Three patients (9\%) were initially observed with serial MRI for a median 16 months (range 11-66 months) prior to treatment. These 3 cases exhibited radiographic evidence of tumor progression before radiosurgery was pursued. There were 25 patients treated with GKRS following previous resection. Of these 25 patients, 11 (44\%) had documented radiographic recurrence, 4 patients $(16 \%)$ had a known area of residual tumor that subsequently grew, and 1 patient (4\%) had residual tumor without documented growth but elected to undergo treatment to prevent progression. Staged GKRS following intentional STR was performed in 6 patients (24\%), all of whom underwent GKRS 4 months following surgery. This 4-month interval was chosen to assist in differentiating early postoperative enhancement from residual tumor. Among those with radiographic recurrence following prior resection, the time of GKRS treatment was highly variable (range 15-206 months).

\section{Patients With More Than 10 Years of Radiographic Follow-Up}

There were 16 patients with more than 10 years of serial MRI data following GKRS (median follow-up 162.5 months). This subset exhibited similar demographics to the overall population (median age 56.5 years; median tumor size $6970 \mathrm{~mm}^{3} ; 50 \%$ of patients treated with GKRS after prior surgery). Among this group, there were 3 with radiographic tumor progression and none with clinical tu-

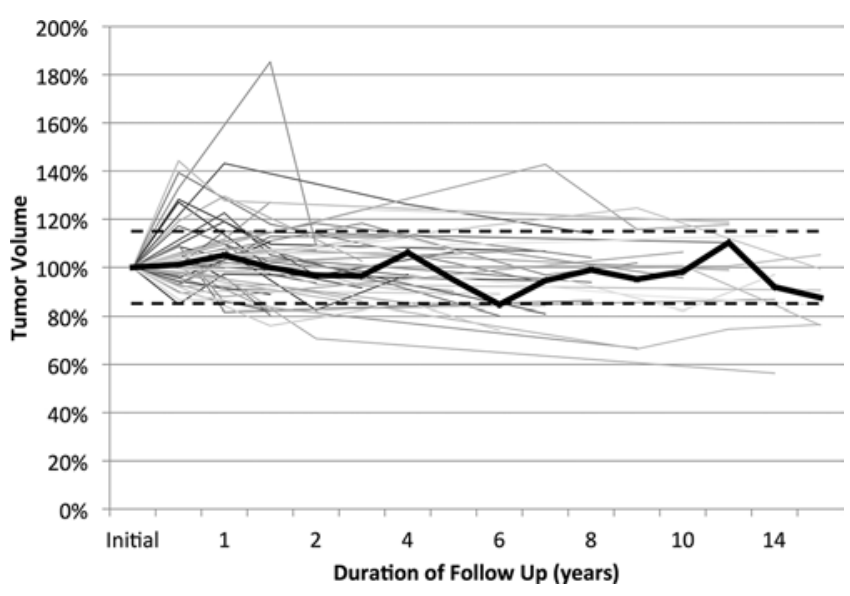

FIG. 3. Graph of individual tumor volumes over time. Median is represented by the thick black line; dashed lines indicate $\pm 15 \%$ volumetric change.

mor progression. Those who progressed exhibited volumetric tumor growth of $17.8 \%, 18.7 \%$, and $19.1 \%$. None developed a new, documented lower cranial neuropathy or facial nerve weakness. Six tumors exhibited a decrease in volume of more than $10 \%$. Four tumors demonstrated a volume increase or decrease of less than $5 \%$ over the follow-up period. The remaining 3 tumors exhibited growth of less than $15 \%$; consistent with the study methodology, these did not meet criteria for radiographic progression.

\section{Familial Paraganglioma Syndrome}

There were 7 patients in the current series with multiple paragangliomas consistent with familial paraganglioma syndrome. The median age at the time of GKRS was 41 years. One patient underwent intentional STR to protect lower $\mathrm{CN}$ function and subsequent GKRS to the residual tumor approximately 4 months later. The remaining 6 patients were treated primarily with GKRS with no evidence of radiographic or clinical progression. The median duration of radiographic follow-up for this subset of patients was 62 months.

\section{Cranial Neuropathy}

Of the 60 patients in this series, $2(3 \%)$ developed documented CN X neuropathy. In 1 case, the patient was initially treated with a marginal dose of $12 \mathrm{~Gy}$, subsequently exhibited tumor progression, and was treated with repeat GKRS to a marginal dose of $14 \mathrm{~Gy}$. While durable tumor control over a 5-year follow-up period was achieved, the patient developed new vocal fold paralysis. The patient's clinical follow-up at our institution was brief due to travel limitations, and therefore neither a laryngoscopic examination confirming vocal fold paralysis nor the date of onset is available. In the second case, ipsilateral vocal fold paralysis, confirmed by laryngoscopy, developed 11 years after GKRS for tumor recurrence following previous resection. There were no cases of new CN XII or VII weakness following GKRS.

\section{Dose to ICA}

The anatomical proximity of most JPs to the ICA sug- 
gests that the cervical $(\mathrm{C}-1)$, petrous $(\mathrm{C}-2)$, and lacerum (C-3) segments may receive radiation during SRS for JP. Moderate and high doses of conventional EBRT to the neck have been associated with atherosclerosis and subsequent transient ischemic attack or stroke. ${ }^{34}$ Coupled with the notion that radiation-induced endothelial damage and intimal fibrosis may be mechanisms for tumor quiescence, one may theorize that these patients may be at greater risk for cerebrovascular sequelae. Thirty-two patients had GammaPlan data available for measurement of radiosurgical dose to the ICA. The median ICA maximum dose was 22.7 Gy (IQR 20.9-25.3 Gy, range 11.2-31.4 Gy). Fortunately, there have been no identified cases of asymptomatic or symptomatic carotid stenosis in this series.

\section{Dose to Cochlea}

Cochlear dose data were available for 26 (43\%) of the 60 patients in this series. The median maximum dose to the cochlear volume was 16.2 Gy (IQR 14-19.1 Gy, range 10.1-26.7 Gy). The median mean dose to the cochlear volume was 8.9 Gy (IQR 7-8.9 Gy, range 4.7-15.9 Gy).

\section{Discussion \\ Overall Tumor Control}

In this report, the authors used 3D volumetric analysis of serial MRI data to demonstrate that excellent tumor control outcomes can be achieved with GKRS for JP. Because only 5 patients experienced radiographic tumor progression, with 1 requiring salvage treatment (i.e., clinical failure), the overall tumor control rate in this series was $91.7 \%$. Progression-free survival was $98 \%$ at 5 years and $94 \%$ at 10 years. These findings are comparable to available radiosurgical series, summarized in Table 4.1,3,8-11, $13-16,22,23,25,28,29,31,33,35,37-40,45$ Nine of these publications report outcomes with more than 4 years of radiographic followup. $9,10,13,16,23,29,36,40,45$ Excluding the present study, the 3 largest series comprise 132,75 , and 58 patients with tumor control rates of $93 \%,{ }^{40} 93.4 \%,{ }^{23}$ and $94.8 \%,{ }^{13}$ respectively, over similar follow-up intervals.

\section{Tumor Volumetry and Definition of Growth}

Linear measurements (with progressive disease based on RECIST [Response Evaluation Criteria In Solid Tumors] criteria, ${ }^{44}$ for example) or volumetric estimation using ellipsoid approximations based on 3 orthogonal measurements or cubed maximal linear diameter may be considered reasonable approaches for the determination of radiographic progression in spherical solid tumors. However, the irregular growth pattern of JP is not compatible with these measurement strategies. This is even more challenging in cases of recurrence or in the presence of postoperative inflammatory or fibrotic changes. We found that using the tumor volume generated with the GammaPlan software likely overestimates actual tumor volume, as the tendency in our practice is to include equivocal areas at the boundary between what is obviously tumor and what is obviously normal tissue in the plan to ensure that the entire tumor is treated. The measurement technique used in this study offers a highly precise and sensitive means of defining tumor boundaries in 3D to objectively assess tumor progression. A report by Varughese et al. supports this measurement strategy with the finding that area-based measurements are more reliable in the detection of smaller volume differences than diameter-based measurements. ${ }^{46}$ In a 2008 study on treatment response criteria for glioma, Sorensen et al. supported the use of volumetric analysis over linear or cross-sectional measurements to increase the sensitivity in detecting tumor volume changes, but acknowledge the challenges in efficiently performing volumetric analysis routinely and determining whether a change in volume is clinically significant. ${ }^{43}$ All scans were analyzed by a single measurer to maintain consistency. As discussed in the Methods section, intrarater correlation was high.

The definition of tumor growth in JP remains undefined in the literature. Snell et al. described the use of dosevolume histograms to estimate measurement error. To ensure a maximum measurement error of $10 \%$, a minimum of 5 axial slices was required to calculate a volume. ${ }^{41} \mathrm{We}$ remained consistent with this protocol and excluded patients with inadequate imaging. Few available series define "growth" beyond simply an increase in tumor size over the follow-up interval. Chen et al. ${ }^{3}$ used $15 \%$ as a cutoff for volumetric growth, but did not enumerate the method of measurement. In the vestibular schwannoma literature, the range of what is considered volumetric growth is quite broad. Harris et al. compared 1D measurements with volumes computed by segmented analysis in patients with vestibular schwannoma related to neurofibromatosis Type 2 . Progressive tumors were considered those with $73 \%$ volume growth, which corresponds to the cube of $20 \%$ linear change. ${ }^{19}$ Kandathil et al. used a cutoff of $20 \%$ for volumetric growth using slice-by-slice volumetric segmentation. ${ }^{26}$ Similarly, Carlson et al. used an ellipsoid approximation and defined a cutoff of $20 \%$ for volumetric growth. ${ }^{2}$ With these studies as the basis for our methodology, 15\% was used as the cutoff for volumetric growth in our series.

\section{Patients With Extended Follow-Up}

In this series, there were 16 patients with more than 10 years of radiographic follow-up (total 218 patient-years). Three of these patients (19\%) were classified as having radiographic tumor progression due to tumor growth over the $15 \%$ cutoff. None of these patients required subsequent treatment intervention for any indication. The only comparable GKRS series with more than 10 years of radiographic follow-up was published by Liscak et al. in 2014. ${ }^{29}$ Their group included 44 patients (albeit with 6 glomus tympanicum tumors) treated to a median marginal dose of $20 \mathrm{~Gy}$ and median maximum dose of 40 Gy. Tumor control was $97.8 \%$ over a median follow-up period of 118 months. The disparity in tumor control rates between the present series and theirs may be related to differences in radiosurgical dose or may be an artifact of measurement methodology, as the assessment of growth or tumor shrinkage was not defined in that study. As all 3 of the patients defined as exhibiting radiographic progression in our series had growth between $15 \%$ and $20 \%$, a less strict definition of tumor growth would change the tumor control rate in this subset considerably. 
TABLE 4. Radiosurgical series for primary and recurrent treatment of JP

\begin{tabular}{|c|c|c|c|c|c|c|c|}
\hline Authors \& Year & $\begin{array}{l}\text { No. of } \\
\text { Pts }\end{array}$ & $\begin{array}{l}\text { Delivery } \\
\text { Method }\end{array}$ & $\begin{array}{l}\text { Marginal } \\
\text { Dose (Gy) }\end{array}$ & $\begin{array}{l}\text { Tumor Control } \\
\text { Rate }(\%)\end{array}$ & $\begin{array}{l}\mathrm{FU} \\
(\mathrm{mos})\end{array}$ & Definition of Progression & Comments \\
\hline Dobberpuhl et al., 2016 & 12 & GKRS & Median 15 & 100.0 & 27.6 & Growth & \\
\hline Ibrahim et al., 2017 & 75 & GKRS & Median 18 & 93.4 & 51.5 & $\begin{array}{l}\text { Volumetric growth using Gamma- } \\
\text { Plan software or linear growth } \\
>2 \mathrm{~mm} \text { in any dimension }\end{array}$ & \\
\hline Schuster et al., 2016 & 14 & LINAC & NA & 92.9 & 31.7 & Growth & \\
\hline El Majdoub et al., 2015 & 27 & LINAC & Median 15 & 100.0 & 129.2 & Growth & \\
\hline $\begin{array}{l}\text { Gandía-González et al., } \\
2014\end{array}$ & 58 & GKRS & Mean 13.6 & 94.8 & 76.6 & Growth & \\
\hline Liscak et al., 2014 & 44 & GKRS & Median 20 & 97.8 & 118 & Growth & 6 glomus tympanicum \\
\hline Hurmuz et al., 2013 & 14 & CyberKnife & $25 \mathrm{~Gy}$ in $5 \mathrm{Fx}$ & 100.0 & 39 & Growth & \\
\hline Sheehan et al., 2012 & 132 & GKRS & Median 15 & 93.0 & 50.5 & Growth (varied) & \\
\hline Lee et al., 2011 & 14 & GKRS & NA & 100.0 & 40.3 & Growth & 3 glomus tympanicum \\
\hline Chen et al., 2010 & 15 & GKRS & Mean 14.6 & 80.0 & 43.2 & $15 \%$ increase in vol & \\
\hline Genç et al., 2010 & 18 & GKRS & Median 15 & 94.4 & 41.5 & $\begin{array}{l}\text { Volumetric growth using radial } \\
\text { ellipse approximation }(V=4 \pi / 3 \\
\left.\times r_{1} \times r_{2} \times r_{3}\right)^{*}\end{array}$ & \\
\hline Ganz \& Abdelkarim, 2009 & 14 & GKRS & Mean 13.6 & 100.0 & 28 & Growth & \\
\hline Miller et al., 2009 & 5 & GKRS & Mean 15 & 100.0 & 29 & Growth & \\
\hline Sharma et al., 2008 & 10 & GKRS & Mean 16.4 & 100.0 & 25.4 & Growth & \\
\hline Bitaraf et al., 2006 & 14 & GKRS & Median 18 & 100.0 & 18.5 & Growth & \\
\hline Feigl \& Horstmann, 2006 & 10 & GKRS & Mean 17 & 100.0 & 33 & Vol reduction $>10 \%$ & \\
\hline Gerosa et al., 2006 & 20 & GKRS & Mean 17.5 & 100.0 & 50.85 & $\begin{array}{l}\text { Volumetric growth using Gamma- } \\
\text { Plan software }\end{array}$ & \\
\hline Poznanovic et al., 2006 & 8 & LINAC & Median 15 & 100.0 & 15.6 & Growth & \\
\hline Varma et al., 2006 & 17 & GKRS & Median 15 & 76.0 & 48 & $\begin{array}{l}\text { Volumetric growth using propri- } \\
\text { etary software }\end{array}$ & \\
\hline Sheehan et al., 2005 & 8 & GKRS & Median 15 & 100.0 & 28 & Growth & \\
\hline Eustacchio et al., 2002 & 19 & GKRS & Median 14 & 94.7 & 86.4 & Growth & \\
\hline Saringer et al., 2001 & 13 & GKRS & NA & 100.0 & 50.4 & Growth & \\
\hline Jordan et al., 2000 & 7 & GKRS & Mean 16.3 & 100.0 & 27 & Growth & \\
\hline Present study & 60 & GKRS & Median 16 & 91.7 & 66 & $\begin{array}{l}\text { Volumetric growth by serial tumor } \\
\text { segmentation }>15 \%\end{array}$ & \\
\hline
\end{tabular}

$\mathrm{FU}=$ follow-up; $\mathrm{NA}=$ not applicable; pts = patients.

${ }^{*} r=$ radius of lesion in each plane.

\section{New or Worsened Cranial Neuropathy}

There were only 2 patients in this series with new or worsened lower cranial neuropathy following GKRS. Both involved CN X that manifested as vocal fold paralysis. Both cases occurred in patients treated with radiosurgery for recurrent disease (1 after prior surgery and 1 after prior GKRS), and both were limited to vocal fold paralysis only. In a meta-analysis of 869 patients with JP treated with surgery, SRS, or a combination, Ivan et al..$^{24}$ reported a pooled estimate of new CN X neuropathy of $9.7 \%$ for patients treated with primary SRS. Combined with data from the present series, we estimate that the actual rate of new CN X neuropathy is low. It is our opinion that the risk of a complete vagal paralysis from radiosurgery is negligible, especially when compared with surgery. This finding has affected our treatment strategy substantially. A large percentage of JPs at our center are treated with primary GKRS. The excep- tions to this may be very young patients with smaller tumors in which complete resection without lower $\mathrm{CN}$ injury is possible, or patients presenting with bulky posterior fossa disease and considerable brainstem compression. In other cases, limited resection of the middle ear component to improve conductive hearing loss and pulsatile tinnitus, followed by GRKS, has been used. When surgery is pursued in patients with large tumors that present with normal lower CN function, we frequently perform intentional STR in tumors that cannot be easily separated from lower CNs to protect their function. Rather than managing these patients expectantly, we have moved toward preemptive GKRS to the residual tumor in most cases, with the knowledge that the risk of lower cranial neuropathy is low.

\section{Hearing Outcomes}

Of great interest to our group is the effect of radiosur- 
gery for JP on hearing. In the subset treated with primary radiosurgery or those with only conductive hearing loss after prior surgery, we are currently evaluating the effect of cochlear dose and pretreatment hearing level on hearing outcomes. These data are still under review and will be reported separately.

\section{Study Limitations}

While the overall number of patients with JP treated using GKRS is relatively large, this study represents a retrospective review dependent on the completeness of the medical record and imaging database. Of the 85 patients with JP treated with radiosurgery at our institution, 25 were excluded because they did not have sufficient serial MRI data available for 3D volumetric analysis. Very few patients in the series demonstrated tumor progression following treatment $(n=5)$, which limits our ability to perform subgroup statistical analysis to detect risk factors for progression, based on established statistical literature. ${ }^{18}$ Furthermore, a recent series of 12 observed, untreated JPs published by Carlson et al. demonstrates that up to 58\% exhibit no growth over a median follow-up of 7.2 years, where "no growth" was defined as an increase in tumor volume of less than $20 \%$ on serial imaging. ${ }^{2}$ In an era of increasing conservatism, it may be reasonable to observe select newly diagnosed JPs to better estimate radiographic or clinical progression to guide treatment decisions. In most cases, however, the fear of impending loss of lower $\mathrm{CN}$ function often drives the decision to intervene. A prospective trial comparing growth rates of observed tumors to those that have undergone GKRS is not presently available. In addition, it is important to recognize that the volumetric analysis method used in this report is intended to provide a means of assessing tumor response to GKRS. The clinical assessment of disease progression takes into account multiple patient and tumor factors, supporting the discrepancy between the number of patients who require additional treatment (1 patient in this series) and those who only exhibit radiographic tumor growth (5 patients in this series). Finally, clinical follow-up is limited in some patients. Many patients treated at our institution travel a great distance for treatment and limit follow-up to submission of serial MRI only, provided they are neurologically stable.

\section{Conclusions}

SRS for JP in the primary, combined modality, or recurrent setting offers excellent tumor control with minimal risk to $\mathrm{CN}$ function. Radiographic progression, when present, occurs late in the course of follow-up and emphasizes the importance of long-term surveillance. However, clinical progression necessitating treatment intervention is rare. These findings support the practice of using primary SRS or adjuvant SRS following function-preserving STR. Volumetric tumor analysis using a slice-by-slice integral measurement technique offers a precise means of quantifying tumor growth for irregular tumor geometries.

\section{References}

1. Bitaraf MA, Alikhani M, Tahsili-Fahadan P, Motiei-Langroudi R, Zahiri A, Allahverdi M, et al: Radiosurgery for glomus jugulare tumors: experience treating 16 patients in Iran. J Neurosurg 105 Suppl:168-174, 2006

2. Carlson ML, Sweeney AD, Wanna GB, Netterville JL, Haynes DS: Natural history of glomus jugulare: a review of 16 tumors managed with primary observation. Otolaryngol Head Neck Surg 152:98-105, 2015

3. Chen PG, Nguyen JH, Payne SC, Sheehan JP, Hashisaki GT: Treatment of glomus jugulare tumors with gamma knife radiosurgery. Laryngoscope 120:1856-1862, 2010

4. Cole JM, Beiler D: Long-term results of treatment for glomus jugulare and glomus vagale tumors with radiotherapy. Laryngoscope 104:1461-1465, 1994

5. Combs SE, Salehi-Allameh B, Habermehl D, Kessel KA, Welzel T, Debus J: Clinical response and tumor control based on long-term follow-up and patient-reported outcomes in patients with chemodectomas of the skull base and head and neck region treated with highly conformal radiation therapy. Head Neck 36:22-27, 2014

6. Cummings BJ, Beale FA, Garrett PG, Harwood AR, Keane TJ, Payne DG, et al: The treatment of glomus tumors in the temporal bone by megavoltage radiation. Cancer 53:26352640, 1984

7. de Jong AL, Coker NJ, Jenkins HA, Goepfert H, Alford BR: Radiation therapy in the management of paragangliomas of the temporal bone. Am J Otol 16:283-289, 1995

8. Dobberpuhl MR, Maxwell S, Feddock J, St Clair W, Bush ML: Treatment outcomes for single modality management of glomus jugulare tumors with stereotactic radiosurgery. Otol Neurotol 37:1406-1410, 2016

9. El Majdoub F, Hunsche S, Igressa A, Kocher M, Sturm V, Maarouf M: Stereotactic LINAC-radiosurgery for glomus jugulare tumors: a long-term follow-up of 27 patients. PLoS One 10:e0129057, 2015

10. Eustacchio S, Trummer M, Unger F, Schröttner O, Sutter B, Pendl G: The role of Gamma Knife radiosurgery in the management of glomus jugular tumours. Acta Neurochir Suppl 84:91-97, 2002

11. Feigl GC, Horstmann GA: Intracranial glomus jugulare tumors: volume reduction with Gamma Knife surgery. J Neurosurg 105 Suppl:161-167, 2006

12. Foote RL, Pollock BE, Gorman DA, Schomberg PJ, Stafford SL, Link MJ, et al: Glomus jugulare tumor: tumor control and complications after stereotactic radiosurgery. Head Neck 24:332-339, 2002

13. Gandía-González ML, Kusak ME, Moreno NM, Sárraga JG, Rey G, Álvarez RM: Jugulotympanic paragangliomas treated with Gamma Knife radiosurgery: a single-center review of 58 cases. J Neurosurg 121:1158-1165, 2014

14. Ganz JC, Abdelkarim K: Glomus jugulare tumours: certain clinical and radiological aspects observed following Gamma Knife radiosurgery. Acta Neurochir (Wien) 151:423-426, 2009

15. Genç A, Bicer A, Abacioglu U, Peker S, Pamir MN, Kilic T: Gamma knife radiosurgery for the treatment of glomus jugulare tumors. J Neurooncol 97:101-108, 2010

16. Gerosa M, Visca A, Rizzo P, Foroni R, Nicolato A, Bricolo A: Glomus jugulare tumors: the option of gamma knife radiosurgery. Neurosurgery 59:561-569, 2006

17. Gilbo P, Morris CG, Amdur RJ, Werning JW, Dziegielewski PT, Kirwan J, et al: Radiotherapy for benign head and neck paragangliomas: a 45-year experience. Cancer 120:37383743, 2014

18. Harrell FE Jr, Lee KL, Califf RM, Pryor DB, Rosati RA: Regression modelling strategies for improved prognostic prediction. Stat Med 3:143-152, 1984

19. Harris GJ, Plotkin SR, Maccollin M, Bhat S, Urban T, Lev $\mathrm{MH}$, et al: Three-dimensional volumetrics for tracking vestibular schwannoma growth in neurofibromatosis type II. Neurosurgery 62:1314-1320, 2008 
20. Hinerman RW, Mendenhall WM, Amdur RJ, Stringer SP, Antonelli PJ, Cassisi NJ: Definitive radiotherapy in the management of chemodectomas arising in the temporal bone, carotid body, and glomus vagale. Head Neck 23:363-371, 2001

21. House JW, Brackmann DE: Facial nerve grading system. Otolaryngol Head Neck Surg 93:146-147, 1985

22. Hurmuz P, Cengiz M, Ozyigit G, Yazici G, Akyol F, Yildiz F, et al: Robotic stereotactic radiosurgery in patients with unresectable glomus jugulare tumors. Technol Cancer Res Treat 12:109-113, 2013

23. Ibrahim R, Ammori MB, Yianni J, Grainger A, Rowe J, Radatz M: Gamma Knife radiosurgery for glomus jugulare tumors: a single-center series of 75 cases. J Neurosurg 126:1488-1497, 2017

24. Ivan ME, Sughrue ME, Clark AJ, Kane AJ, Aranda D, Barani IJ, et al: A meta-analysis of tumor control rates and treatment-related morbidity for patients with glomus jugulare tumors. J Neurosurg 114:1299-1305, 2011

25. Jordan JA, Roland PS, McManus C, Weiner RL, Giller CA: Stereotactic radiosurgery for glomus jugulare tumors. Laryngoscope 110:35-38, 2000

26. Kandathil CK, Cunnane ME, McKenna MJ, Curtin HD, Stankovic KM: Correlation between aspirin intake and reduced growth of human vestibular schwannoma: volumetric analysis. Otol Neurotol 37:1428-1434, 2016

27. Konefal JB, Pilepich MV, Spector GJ, Perez CA: Radiation therapy in the treatment of chemodectomas. Laryngoscope 97:1331-1335, 1987

28. Lee CC, Pan DH, Wu JC, Chung WY, Wu HM, Yang HC, et al: Gamma knife radiosurgery for glomus jugulare and tympanicum. Stereotact Funct Neurosurg 89:291-298, 2011

29. Liscak R, Urgosik D, Chytka T, Simonova G, Novotny J Jr, Vymazal J, et al: Leksell Gamma Knife radiosurgery of the jugulotympanic glomus tumor: long-term results. J Neurosurg 121 Suppl:198-202, 2014

30. McCaffrey TV, Meyer FB, Michels VV, Piepgras DG, Marion MS: Familial paragangliomas of the head and neck. Arch Otolaryngol Head Neck Surg 120:1211-1216, 1994

31. Miller JP, Semaan MT, Maciunas RJ, Einstein DB, Megerian CA: Radiosurgery for glomus jugulare tumors. Otolaryngol Clin North Am 42:689-706, 2009

32. Pollock BE: Stereotactic radiosurgery in patients with glomus jugulare tumors. Neurosurg Focus 17(2):E10, 2004

33. Poznanovic SA, Cass SP, Kavanagh BD: Short-term tumor control and acute toxicity after stereotactic radiosurgery for glomus jugulare tumors. Otolaryngol Head Neck Surg 134:437-442, 2006

34. Ravin RA, Gottlieb A, Pasternac K, Cayne N, Schneider D, Krishnan P, et al: Carotid artery stenting may be performed safely in patients with radiation therapy-associated carotid stenosis without increased restenosis or target lesion revascularization. J Vasc Surg 62:624-630, 2015

35. Saringer W, Khayal H, Ertl A, Schoeggl A, Kitz K: Efficiency of gamma knife radiosurgery in the treatment of glomus jugulare tumors. Minim Invasive Neurosurg 44:141-146, 2001

36. Saringer W, Kitz K, Czerny C, Kornfehl J, Gstöttner W, Matula $\mathrm{C}$, et al: Paragangliomas of the temporal bone: results of different treatment modalities in 53 patients. Acta Neurochir (Wien) 144:1255-1264, 2002

37. Schuster D, Sweeney AD, Stavas MJ, Tawfik KY, Attia A, Cmelak AJ, et al: Initial radiographic tumor control is similar following single or multi-fractionated stereotactic radiosur- gery for jugular paragangliomas. Am J Otolaryngol 37:255258,2016

38. Sharma MS, Gupta A, Kale SS, Agrawal D, Mahapatra AK, Sharma BS: Gamma knife radiosurgery for glomus jugulare tumors: therapeutic advantages of minimalism in the skull base. Neurol India 56:57-61, 2008

39. Sheehan J, Kondziolka D, Flickinger J, Lunsford LD: Gamma knife surgery for glomus jugulare tumors: an intermediate report on efficacy and safety. J Neurosurg 102 Suppl:241-246, 2005

40. Sheehan JP, Tanaka S, Link MJ, Pollock BE, Kondziolka D, Mathieu D, et al: Gamma Knife surgery for the management of glomus tumors: a multicenter study. J Neurosurg 117:246-254, 2012

41. Snell JW, Sheehan J, Stroila M, Steiner L: Assessment of imaging studies used with radiosurgery: a volumetric algorithm and an estimation of its error. Technical note. J Neurosurg 104:157-162, 2006

42. Sobol SM, Dailey JC: Familial multiple cervical paragangliomas: report of a kindred and review of the literature. Otolaryngol Head Neck Surg 102:382-390, 1990

43. Sorensen AG, Batchelor TT, Wen PY, Zhang WT, Jain RK: Response criteria for glioma. Nat Clin Pract Oncol 5:634644, 2008

44. Therasse P, Arbuck SG, Eisenhauer EA, Wanders J, Kaplan RS, Rubinstein L, et al: New guidelines to evaluate the response to treatment in solid tumors. European Organization for Research and Treatment of Cancer, National Cancer Institute of the United States, National Cancer Institute of Canada. J Natl Cancer Inst 92:205-216, 2000

45. Varma A, Nathoo N, Neyman G, Suh JH, Ross J, Park J, et al: Gamma knife radiosurgery for glomus jugulare tumors: volumetric analysis in 17 patients. Neurosurgery 59:1030-1036, 2006

46. Varughese JK, Wentzel-Larsen T, Vassbotn F, Moen G, Lund-Johansen M: Analysis of vestibular schwannoma size in multiple dimensions: a comparative cohort study of different measurement techniques. Clin Otolaryngol 35:97-103, 2010

47. Wanna GB, Sweeney AD, Haynes DS, Carlson ML: Contemporary management of jugular paragangliomas. Otolaryngol Clin North Am 48:331-341, 2015

\section{Disclosures}

The authors report no conflict of interest concerning the materials or methods used in this study or the findings specified in this paper.

\section{Author Contributions}

Conception and design: Carlson, Patel, Pollock, Link. Acquisition of data: Patel, Pollock. Analysis and interpretation of data: Carlson, Patel, Lohse, Link. Drafting the article: Carlson, Patel, Lohse, Link. Critically revising the article: Carlson, Patel, Pollock, Driscoll, Neff, Foote, Link. Reviewed submitted version of manuscript: all authors. Approved the final version of the manuscript on behalf of all authors: Carlson. Statistical analysis: Patel, Lohse. Study supervision: Carlson, Link.

\section{Correspondence}

Matthew L. Carlson: Mayo Clinic, Rochester, MN. carlson. matthew@mayo.edu. 